\title{
Biochemical and dietary indicators among vegetarians and non-vegetarians: findings from a cross sectional study in rural Bangladesh
}

\author{
Fahmida Dil Farzana ${ }^{1}$, Shahnawaz Ahmed ${ }^{1}$, Farzana Ferdous ${ }^{1}$, Lana Vanderlee ${ }^{2}$, \\ Soroar Hossain Khan ${ }^{1}$, Anjan Kumar Roy ${ }^{3}$, Mohammod Jobayer Chisti ${ }^{1}$, \\ Abu Syed Golam Faruque ${ }^{1}$, Sumon Kumar Das ${ }^{1, *}$ \\ ${ }^{1}$ Centre for Nutrition and Food Security, International Centre for Diarrhoeal Disease Research, Bangladesh, Dhaka, Bangladesh \\ ${ }^{2}$ School of Public Health and Health Systems, University of Waterloo, Waterloo, Canada \\ ${ }^{3}$ Centre for Vaccine Science, Centre for Nutrition and Food Security, International Centre for Diarrhoeal Disease Research, Bangladesh, \\ Dhaka, Bangladesh
}

\begin{abstract}
Email address:
fahmidaf@icddrb.org (F. D. Farzana),shahnawz@icddrb.org (S. Ahmed), farzanaf@icddrb.org (F. Ferdous), 12vander@mailservices.uwaterloo.ca (L. Vanderlee), soroar@icddrb.org (S. H. Khan), anjan@icddrb.org (A. K. Roy), chisti@icddrb.org (M. J. Chisti), gfaruque@icddrb.org (A. S. G. Faruque), sumon@icddrb.org (S. K. Das)
\end{abstract}

\section{To cite this article:}

Fahmida Dil Farzana, Shahnawaz Ahmed, Farzana Ferdous, Lana Vanderlee, Soroar Hossain Khan, Anjan Kumar Roy, Mohammod Jobayer Chisti, Abu Syed Golam Faruque, Sumon Kumar Das. Biochemical and Dietary Indicators among Vegetarians and NonVegetarians: Findings from a Cross Sectional Study in Rural Bangladesh. International Journal of Nutrition and Food Sciences. Vol. 2, No. 3, 2013, pp. 130-136. doi: 10.11648/j.ijnfs.20130203.16

\begin{abstract}
Well-planned vegetarian diet provides numerous health benefits and is appropriate for all stages of life cycle. On the other hand, vegetarians often suffer from lower level or are deficient with different micro as well as macro nutrients. This ultimately led the study to determine and compare dietary intake and biochemical concentration of macro and micronutrients of vegetarian and non-vegetarian individuals. This cross sectional study was conducted during AprilSeptember 2010. Sixty six vegetarian and 66 non-vegetarian healthy adult aged 40 years and above were enrolled from the Demographic Surveillance System area of Mirzapur sub-district under Tangail district of rural Bangladesh. Five milliliters $(5.0 \mathrm{ml})$ of venous blood was collected for the biochemical analysis. Dietary assessment involved 24-hour food recall method. This study revealed that, vegetarians had similar BMI compared to non-vegetarians. Biochemical analysis unveiled mean level of folic acid to be significantly higher $(23.1 \pm 12.7$ vs. $16.9 \pm 8.8, \mathrm{p}=0.002)$ and mean level of vitamin $\mathrm{B} 12$ $(134.9 \pm 46.2$ vs. $217.6 \pm 95.6, \mathrm{p}<0.001)$ to be significantly lower in vegetarians compared to non-vegetarians. From dietary history, mean calorie intake ( $1671.9 \pm 788.5 \mathrm{kcal}$ vs. $1908.9 \pm 574.5 \mathrm{kcal}, \mathrm{p}=0.013)$ and macronutrient (median value; per day) consumption was found lower [carbohydrate (334.5 gm vs. $385.1 \mathrm{gm}, \mathrm{p}=0.048)$; protein ( $34.9 \mathrm{gm}$ vs. $53.9 \mathrm{gm}, \mathrm{p}<0.001$ ); fat ( $7.7 \mathrm{gm}$ vs. $12.7 \mathrm{gm}, \mathrm{p}=0.020)$ ] in non-vegetarian group compared to vegetarian individuals. In multivariate analysis of biochemical indicators, individuals being vegetarian impacted on decreased level of vitamin B12 (74.02 pmol/L) and increased level of folic acid $(6.34 \mathrm{nmol} / \mathrm{L})$. Multivariate analysis of dietary indicators revealed, individuals being vegetarian has an impact on decreased intake of protein $(7.85 \mathrm{gm})$ and increased intake of log transformed $\beta$-carotene $(0.61 \mathrm{mcg})$. These results suggest that, vegetarians in rural Bangladesh have lower intake of protein and micronutrients specially vitamin B 12; but increased intake of folic acid and $\beta$-carotene. Assessment of these outcomes affirms further study.
\end{abstract}

Keywords: Bangladesh, Calorie, Folic Acid, Non-Vegetarian, Vegetarian, Vitamin B-12

\section{Introduction}

During the past decades, knowledge has emerged on the effect of vegetarian diet on nutritional status [1]. There is evidence that well-planned vegetarian diet provides numerous health benefits and is appropriate for all stages of life cycle [2]. Considering health hazards of consumption of protein rich diet deriving from animal sources, proportion of vegetarian or people consuming vegetable 
based diet is increasing globally [3-6]. Global health economy is recently experiencing high burden of chronic diseases as cerebrovascular diseases, cardiovascular disease as well as endocrine disorders like diabetic mellitus with sufferings from malignancy [7-10]. This situation has led the concept of vegetarianism to become much popular not only in developed countries but also in developing one [1113]. On the other hand, vegetarians are often deficient in different macro as well as micro nutrients especially vitamin B12 and the sufficiency mainly found in animal food sources [14]. Chronic deficiency often manifests with clinical syndrome and need appropriate medication or replacement through properly planned diet or vitamin supplements. In most of the cases, inadequate knowledge about the food sources of such essential micronutrient as well as the availability of the particular food items might be the underlying causes.

Like other developing countries, Bangladesh is now burdened with pretty high rate of chronic diseases among the general population $[15,16]$. As a result, vegetarian diet is now becoming familiar and considered as healthy diet as appropriate medication. A very recent study revealed favorable lipoprotein status among rural Bangladeshi vegetarians indicating the importance of consumption of vegetable based diet [17]. While looking for studies on vegetarian and non-vegetarian individuals conducted in our country context, lack of information approached; particularly, dietary consumption of micronutrient with its availability in the serum concentration. So, it was a sine qua non to address the existing knowledge gap on this. Thus, the present study aimed to determine the availability of macro and micro nutrients in diet as well as in serum concentration among healthy adult vegetarians; and at the same time, compare these values with healthy adults consuming a regular diet.

\section{Methodology}

\subsection{Description of the Study area}

Participants were selected from those living in the Demographic Surveillance System (DSS) area of Mirzapur sub-district under Tangail district of rural Bangladesh. This DSS was established by the International Centre for Diarrhoeal Disease Research, Bangladesh (icddr,b) with a population of over 255 thousands to collect longitudinal information on their vital events such as birth, death, marriage, and migration. About $30 \%$ of the DSS population are 40 years or older, and all have unique demographic surveillance identity. Kumudini Hospital, a 750-bed tertiary level hospital serves the DSS residents along with individuals reporting from other areas of rural Bangladesh.

\subsection{Study Design}

This survey was conducted as cross-sectional, from April to September 2010.

\subsection{Selection Criteria}

\subsubsection{Inclusion Criteria}

Vegetarian were defined as individuals consuming vegetable-based diets only, devoid of any animal protein for more than last 5 years. At the time of initial screening, it was a bit difficult to get a vegetarian in rural Bangladesh. However, those who were identified, majority (92\%) gave a history of being on vegetarian diet for more than five years. People on the usual Bangladeshi diets, including animal protein, were considered as non-vegetarian controls. Trained research assistants screened vegetarians through house to house visit of randomly selected villages within the DSS area. After initial screening at the field, all interested participants were invited to come to Kumudini Hospital for final selection.

\subsubsection{Exclusion Criteria}

Potential individuals were asked for current or previous history of respiratory diseases, chronic illnesses such as hypertension, diabetes mellitus, and ischemic heart disease and were excluded during initial screening.

\subsection{Sample Size}

Total 100 adult vegetarians aged 40 years and above were identified and invited to participate; 66 were enrolled meeting the inclusion criteria and willing to participate upon providing written consent. Equal number $(n=66)$ of adult non-vegetarians of same age stratum \pm 4 years and sex from neighborhood were included following same procedure. Moreover, participants' optimal hepatic function (alanine aminotransferase) and renal function (serum creatinine) were also assessed.

\subsection{Ethical Consideration}

The study objectives and its methods, and its risks and benefits, as well as their rights as participants and confidentiality of information taken from them were described to each of the participants before their final enrollment after signing informed consent form.

\subsection{Anthropometric Measurement and Blood Sampling}

Height and weight of all the participants were measured and Body Mass Index (BMI) was calculated as $\left(\mathrm{kg} / \mathrm{m}^{2}\right)$. Five milliliters $(5.0 \mathrm{ml})$ of venous blood was collected. Blood pressure was measured by sphygmomanometer. For hemoglobin estimation, $0.5 \mathrm{ml}$ blood was put into an Eppendorf tube containing Ethylenediaminetetraacetic acid (EDTA) and rest was poured into a venoject tube for serum separation. All specimens were transported immediately to the Nutritional Biochemistry Laboratory of icddr,b in Dhaka for the biochemical analyses of: hemoglobin [18], serum uric acid (Roche), alanin aminotransferase (ALT) [19], calcium [20], albumin [21], retinol [22], vitamin B12 (IMMULITE), folic acid (IMMULITE), serum creatinine [23] and zinc [24] following standard laboratory procedures. 
Fasting blood sugar (FBS) was determined by a bedside glucometer (ACCU-CHEK, Active, Roche) at the time of blood collection to exclude any diabetic and pre-diabetic status.

\subsection{Dietary Intake Data}

Dietary history was taken using the 24-hour recall method. This widely-used method involves asking subjects to recall and describe all intakes of foods and drinks in the previous 24 hours. While recording dietary history in our study, the interviewers probed respondents for information on food items, portion-size, recipe ingredients and cooking methods. Pre standardized food models and household measuring instruments were used for increasing the accuracy of portion-size. The tools that were used for datacollection included a plate, a bowl, a cup, a glass, and six different types of spoons [ 1 rice, 2 curry, 1 dal (lentil soup), 1 table and 1 tea spoon]. Then the portion-size was converted into weight (gram). The nutrient contents as well as the calorie values of each food were calculated thereafter using standard references on food value [25]. Using MSExcel, a unique excel spreadsheet was developed for the calculation of recall data. This sheet was comprised of standard calorie value of food items consumed by the respondents. Data were entered as proportion of the prestandardized tools for each food item. Total calorie/person/day was automatically generated by using mathematical formulas for calculations. Deshio Khadder Pushtiman (INFS, Dhaka University) and Nutritive value of
Indian Foods (National Institute of Nutrition, Indian council of Medical Research) served as standard references for calculating values of each nutrient. Each calculated variable was then transferred to SPSS database for subsequent analysis. Food Consumption Score (FCS) was also taken as a proxy indicator to get the pattern of food consumption within the family; it is a composite score based on dietary diversity, food frequency and relative nutritional importance of different food groups [26].

\subsection{Data Analysis}

Data analysis was done using Statistical Package for Social Sciences (SPSS) Windows (Version 15.2; Chicago, IL) and Epi Info (Version 6.0, USD, Stone Mountain, GA). We performed Student's t test for all continuous variables and in case of non-normal distribution of data, equivalent non-parametric test (Mann Whitney test) was applied. Moreover, log transformation was also done for variables with skewed distribution. Probability of $<0.05$ was considered to be statistically significant and their $95 \%$ confidence intervals (CI) were also equated. Finally, regression method was performed for vitamins and minerals in blood or serum and dietary composition of energy and micronutrient as dependent variables to find any association with status (vegetarian vs. non-vegetarian) as independent variable and other biochemical markers (ALT), host characteristics (age, sex, BMI), personal habit (smoking habit) and FCS as confounders that might influence the outcome.

Table 1. Estimation of biochemical markers among study participants

\begin{tabular}{lll}
\hline Indicator $($ mean \pm SD) & Vegetarian & Non- vegetarian \\
\hline Hemoglobin $(\mathrm{g} / \mathrm{dL})$ & $12.41 \pm 1.77$ & $12.74 \pm 1.39$ \\
Alanine aminotransferase $(\mathrm{ALT})(\mathrm{U} / \mathrm{L})$ & $19.26 \pm 9.50$ & $18.49 \pm 11.26$ \\
Retinol $(\mu \mathrm{g} / \mathrm{dL})$ & $37.85 \pm 10.09$ & $39.48 \pm 11.36$ \\
Calcium $(\mathrm{mg} / \mathrm{dL})$ & $9.23 \pm 0.43$ & $9.31 \pm 0.49$ \\
Albumin $(\mathrm{g} / \mathrm{dL})$ & $4.26 \pm 0.28$ & $4.31 \pm 0.28$ \\
Folic acid $(\mathrm{nmol} / \mathrm{L})$ & $23.14 \pm 12.74$ & $16.86 \pm 8.75$ \\
Vitamin B12 $(\mathrm{pmol} / \mathrm{L})$ & $134.97 \pm 46.21$ & $217.56 \pm 95.64$ \\
Serum creatinine $(\mu \mathrm{mol} / \mathrm{L})$ & $60.76 \pm 18.72$ & 0.410 \\
Uric acid $(\mu \mathrm{mol} / \mathrm{L})$ & $265.97 \pm 79.15$ & $271.02 \pm 70.64$ \\
Zinc $(\mathrm{mg} / \mathrm{L})$ & $0.93 \pm 0.19$ & $0.89 \pm 0.20$ \\
Fasting blood sugar $(\mathrm{mmol} / \mathrm{L})$ & $5.77 \pm 1.15$ & $5.95 \pm 1.96$ \\
Body Mass Index $(\mathrm{BMI})$ & $20.23 \pm 3.25$ & 0.002 \\
Food Consumption Score $(\mathrm{FCS})$ & $53 \pm 16$ & $20.34 \pm 2.92$ \\
\hline
\end{tabular}

\section{Results}

Of the enrolled participants $(n=132)$, half were vegetarian (30 males and 36 females) and other half were non-vegetarian ( 30 males and 36 females). The mean age of the vegetarians and non-vegetarians were 58 years $(\mathrm{SD} ; \pm 12)$ (age span; 41 to 93 years) and 57 years $(\mathrm{SD} ; \pm 9)$ (age span; 43 to 85 years) respectively. All study participants were found to be identical with optimal renal (serum creatinine) and hepatic functions (ALT) and they also had similar BMI. At least $24 \%$ respondents in both the groups gave a history of smoking $(25 \%$ in non-vegetarian group).

In vegetarian group, mean value of folic acid was significantly higher and mean value of vitamin B12 was significantly lower compared to non-vegetarian group. Other biochemical markers such as blood hemoglobin, serum retinol, calcium, albumin, uric acid, zinc, including fasting blood sugar remained similar in both the groups (Table 1). However, families belonging to the vegetarians had lower FCS compared to non-vegetarians. 
Mean daily consumption of macronutrients (carbohydrate, protein and fat) was lower in vegetarian group. As a result, vegetarians had lower daily calorie intake. However, moisture content and micronutrients included in the consumed food such as iron, zinc, $\beta$ carotene and phytate were similar in case of both the groups (Table 2).

In univariate analysis of biochemical markers, only folic acid and zinc was found to be positively impacted being vegetarian; however, all the other variables were found to be negative. In multivariate analysis, considering the vitamins and micronutrients separately as dependent variable, hemoglobin, retinol, folic acid, serum creatinine, zinc and fasting blood sugar were found to be positively associated after adjusting for age, sex, BMI, ALT, FCS and smoking. Individuals being vegetarian impacted on decreased level of vitamin B12 (74.02 pmol/1) and increased level of folic acid (6.34 nmol/L) (Table 3).

Table 2. Indicators extracted from dietary sources among study participants

\begin{tabular}{lll}
\hline Indicator & Vegetarian $(\mathbf{n}=\mathbf{6 6})$ & Non- vegetarian $(\mathbf{n}=\mathbf{6 6})$ \\
\hline Energy (mean \pm SD) & $1671.90 \pm 788.45$ & $1908.93 \pm 574.49$ \\
Protein (Median) & 37.90 & 53.94 \\
Carbohydrate (Median) & 334.50 & 385.10 \\
Fat (gm) (Median) & 7.74 & 12.70 \\
Moisture (gm) (Median) & 351.93 & 372.62 \\
Iron (mg) (Median) & 10.94 & 13.18 \\
Zinc (mg) (Median) & 10.93 & 10.78 \\
Log transformed $\beta$-carotene (mcg) (mean \pm SD) & $8.02 \pm 1.27$ & 0.048 \\
Phytate (mg) (Median) & 317.40 & 0.020 \\
\hline
\end{tabular}

Table 3. Unadjusted and adjusted mean difference (95\% confidence interval) of different biochemical markers

\begin{tabular}{lll}
\hline Indicator & $\begin{array}{l}\text { Unadjusted mean difference } \\
\mathbf{9 5 \%} \text { CI) }\end{array}$ & $\begin{array}{l}\text { Adjusted mean difference } \\
(\mathbf{9 5 \%} \text { CI) }\end{array}$ \\
\hline Hemoglobin $(\mathrm{g} / \mathrm{dL})$ & $-0.33(-0.95,0.29)$ & $0.20(-0.38,0.79)$ \\
Retinol $(\mu \mathrm{g} / \mathrm{dL})$ & $-1.64(-5.55,2.28)$ & $0.04(-4.05,4.13)$ \\
Calcium $(\mathrm{mg} / \mathrm{dL})$ & $-0.08(-0.25,0.08)$ & $-0.06(-0.24,0.12)$ \\
Albumin $(\mathrm{g} / \mathrm{dL})$ & $-0.05(-0.15,0.05)$ & $-0.02(-0.13,0.08)$ \\
Folic acid $(\mathrm{nmol} / \mathrm{L})$ & $6.28(2.31,10.26)$ & $6.34(2.29,10.39)^{*}$ \\
Vitamin B12 $(\mathrm{pmol} / \mathrm{L})$ & $-82.59(-109.79,-55.39)$ & $-74.02(-102.69,-45.34)^{*}$ \\
Serum creatinine $(\mu \mathrm{mol} / \mathrm{L})$ & $-0.28(-6.32,5.75)$ & $0.81(-4.35,5.96)$ \\
Uric acid $(\mu \mathrm{mol} / \mathrm{L})$ & $-5.05(-32.30,22.20)$ & $-2.70(-25.31,19.91)$ \\
Zinc $(\mathrm{mg} / \mathrm{L})$ & $0.04(-0.03,0.12)$ & $0.05(-0.02,0.13)$ \\
Fasting blood sugar $(\mathrm{mmol} / \mathrm{L})$ & $-0.18(-0.77,0.40)$ & $0.16(-0.45,0.76)$ \\
\hline
\end{tabular}

Outcome variables: Hemoglobin, retinol, calcium, albumin, folic acid, vitamin B12, serum creatinine, uric acid.

Main exposure: status (vegetarian vs. non-vegetarian) non-vegetarian as reference category.

Adjusted factors: age, sex, body mass index (BMI), alanin aminotransferase (ALT), food consumption score (FCS), smoking.

*Values are significant at 5\% level.

In dietary indicators, univariate analysis showed iron, zinc, and $\log$ transformed $\beta$-carotene to be positively impacted being vegetarian; however, other indicators (energy, carbohydrate, protein, fat, and phytate) were found to be negatively impacted. In multivariate analysis, energy, carbohydrate, protein, fat, and phytate were found to be negatively associated after adjusting for age, sex, BMI and FCS. Moisture, iron, zinc, and log transformed $\beta$-carotene were found positively correlated. Individuals being vegetarian impacted on decreased intake of protein $(7.85$ gm) and increased level of log transformed $\beta$-carotene (0.61 mcg) (Table 4).

\section{Discussion}

In the present study, the important observation between vegetarian and non-vegetarian groups was similar level of nutrients in serum as well as in food (consumed in last 24 hours); exception was noticed in protein intake, vitamin B12, folic acid and log transformed $\beta$-carotene. Vegetables and fruits produce minimum energy after being metabolized because of high water and low fat content [27]. As these are the major foods for the vegetarians to thrive on, that explains why the daily calorie intake of the vegetarians was found lower compared to the non-vegetarians.

Though study groups significantly differed in energy intake, their mean BMI was identical. This can be interpreted as the individuals from both the groups were getting nutrients from dietary sources in adequate and equal proportion. A general concept of energy generation is, a

certain amount of food stuff produces a definite amount of energy. Apart from the theoretical overview, another point to be concerned about, whether that certain amount of food particle can yield certain proportion of energy after being metabolized [28]. The respondents probably had chronic disease, or any malfunction of the intestine which impeded the nutrients to be utilized fully/properly. Vegetarians consumed milk $(n=22)$ with their regular diet which is indeed compatible with the nutrient intake of the non-vegetarian from their food sources (22 individuals consumed milk along other food particles) [29]. Proportion of moisture and phytate were found similar between 
vegetarian and non-vegetarian; probable reason happens to be the consumption pattern. Rural individuals generally have fruits and vegetables grown in their own compound, leading to similar consumption pattern irrespective of status (vegetarian or non-vegetarian) (personal communication; ASG Faruque). Additionally, non-vegetarians do not only thrive on foods from animal sources; rather their intake remains limited or minimum due to high costing of the food particles in the local market. Interestingly, log transformed $\beta$-carotene content was found to be increased in case of vegetarian; this might have happened as yellow, orange, and green leafy vegetables and fruits are the richest source of this micronutrient [30].

Table 4. Unadjusted and adjusted mean difference (95\% confidence interval) of different dietary indicators

\begin{tabular}{lll}
\hline Indicator & $\begin{array}{l}\text { Unadjusted mean difference } \\
\mathbf{9 5 \% ~ C I )}\end{array}$ & $\begin{array}{l}\text { Adjusted mean difference } \\
(\mathbf{9 5 \%} \text { CI) }\end{array}$ \\
\hline Energy $(\mathrm{Kcal})$ & $-237.02(-487.82,13.78)$ & $-127.14(-369.04,114.76)$ \\
Protein (gm) & $-13.51(-21.59,-5.43)$ & $-7.85(-15.20,-0.49) *$ \\
Carbohydrate $(\mathrm{gm})$ & $-37.75(-95.16,19.66)$ & $-22.39(-78.84,34.05)$ \\
Fat (gm) & $-4.43(-8.49,-0.36)$ & $-1.23(-4.96,2.49)$ \\
Moisture (gm) & $-41.91(-119.71,35.88)$ & $8.77(-66.43,83.98)$ \\
Iron (mg) & $0.75(-3.52,5.02)$ & $1.64(-2.75,6.02)$ \\
Zinc (mg) & $0.71(-1.87,3.30)$ & $1.91(-0.69,4.51)$ \\
Log transformed $\beta$-carotene $(\mathrm{mcg})$ & $0.05(0.25,-0.01)$ & $0.61(0.08,1.14) *$ \\
Phytate (mg) & $-24.69(-89.98,40.61)$ & $-6.78(-72.19,58.64)$ \\
\hline
\end{tabular}

Outcome variables: Energy, protein, carbohydrate, fat, moisture, iron, zinc, $\beta$-carotene, phytate.

Main exposure: status (vegetarian vs. non-vegetarian) non-vegetarian as reference category.

Adjusted factors: age, sex, body mass index (BMI), food consumption score (FCS).

*Values are significant at $5 \%$ level

Lower serum level of vitamin B12 and higher level of folic acid in vegetarian group might be correlated with their nature of consumption pattern. Vitamin B12 is naturally found in animal food sources including fish, meat, poultry, egg, milk, and milk product; it is generally not present in plant based foods [14]. On the other hand, folic acid is found in abundance in leafy vegetables [31] and in legumes and in moderate amount in fruits; this explains why the vegetarians compared to non-vegetarians in true sense are getting high amount of folic acid from their dietary sources.

The vegetarians whom we have chosen may not be a vegetarian from the beginning of their life; probably they continued normal diet as non-vegetarians in their early life. Usually vitamin B12 remains stored in the liver in large concentration [32] from which they probably got the supply to continue its function. Symptoms of consuming lower level of vitamin B12 or its deficiency may not appear to be detected clinically. Other co-variates as smoking, that contains nicotine might have significant role in vitamin B12 concentration reported by other studies [33]; served as an adjusted factor in the regression model.

We took 24-hour recall data only for a single day (as a cross sectional observation); which might have not reflected their usual intake. If 24-hour recall data had been taken for multiple days [34], it would have given an average picture of the consumption of calorie as well as the nutrients. We have measured FCS, not to comment on the food security status of the household, but to get the pattern of food consumption within the family for last 7 days. This tended to overcome the limitation of taking the dietary recall data for once when placed in the regression model as an independent variable. However, vegetarians had lower FCS score compared to their counterpart; this once again proved lower intake of protein by the vegetarian.

\section{Strength and Weakness}

We had been careful in identifying two groups of healthy study population by eliminating selection bias to the possible extent. In Bangladesh, true vegetarians are fewer and our sample size was thus smaller, which may have resulted in any statistically non-significant association. This could be one of the limitations of the study due to lack of adequate power. However, with this sample size we could first demonstrate important statistically significant associations which may have meaningful public health implications in Bangladesh. There is evidence suggesting serum vitamin B12 concentrations might not accurately reflect intracellular concentrations [14]. However, icddr,b possess the only laboratory that has ISO Accreditation with maintenance of highest standard which we consider to be one of our strengths. The study was designed as cross sectional and self-reported information of those vegetarians in the rural area was analyzed without any direct observation of dietary intake. Thus, findings might have been influenced and might not generalize the rural population of Bangladesh. We also haven't collected any recent morbidity information of the respondents which might have a substantial impact on the micronutrients. However, the present study findings were mostly similar to those observed by earlier larger studies in other countries [35]. Therefore, it is reasonable to consider that our findings as real and not by chance.

\section{Conclusion and Recommendation}


Daily calorie consumption and macronutrient intake of vegetarian individuals demonstrated lower level compared to non-vegetarians. Mean value of folic acid was significantly higher and mean value of vitamin B12 was significantly lower in vegetarian group compared to nonvegetarians; other biomarkers and dietary indicators remained similar. To make their diet calorie dense, vegetarian individuals should be more careful in choosing their foods which is at the same time balanced, healthy and calorie yielding. If financial situation permits, milk can be added to regular diet as well for continue supply of protein and micronutrients. These recommendations along with our findings may help policy makers to introduce health campaign among mass population to promote health benefits of vegetarian diet; a national guideline can be prepared and these findings can contribute to some extent. Outcomes of this study demands further evaluation to allow large extent research on it.

\section{Acknowledgement}

This research study was funded by Government of the People's Republic of Bangladesh through IHP-HNPRP (grant number GR/00410). icddr,b acknowledges with gratitude the commitment of Government of the People's Republic of Bangladesh to its research efforts. Our earnest thanks to Kumudini Women's Medical College and Hospital for providing us the support to conduct the study. We are also thankful to laboratory personals for biochemical analyses of blood specimens and Bidduth Sarkar and other research personals for screening and specimen collection.

\section{References}

[1] Michalak, J., X. C. Zhang, and F. Jacobi, Vegetarian diet and mental disorders: results from a representative community survey. , International Journal of Behavioral Nutrition and Physical Activity, 9 (2012).

[2] Elmadfa, I. and I. Singer, Vitamin B-12 and homocysteine status among vegetarians: a global perspective, Am J Clin Nutr, 89 (2009) 1693S-1698S.

[3] Craig, W. J., Health effects of vegan diets, Am J Clin Nutr, 89 (2009) 1627S-1633S.

[4] Nakamoto, K., et al., A new Japanese vegetarian food guide, Asia Pac J Public Health, 21 (2009) 160-9.

[5] Dunham, L. and L. M. Kollar, Vegetarian eating for children and adolescents, J Pediatr Health Care, 20 (2006) 27-34.

[6] Povey, R., B. Wellens, and M. Conner, Attitudes towards following meat, vegetarian and vegan diets: an examination of the role of ambivalence, Appetite, 37 (2001) 15-26.

[7] Forouzanfar, M. H., et al., Assessing the global burden of ischemic heart disease, part 2: analytic methods and estimates of the global epidemiology of ischemic heart disease in 2010, Glob Heart, 7 (2012) 331-342.

[8] Murray, C. J., et al., Disability-adjusted life years (DALYs) for 291 diseases and injuries in 21 regions, 1990-2010: a systematic analysis for the Global Burden of Disease Study 2010, Lancet, 380 (2012) 2197-223.

[9] Norrving, B. and B. Kissela, The global burden of stroke and need for a continuum of care, Neurology, 80 (2013) S512.

[10] Song, S. H., Emerging type 2 diabetes in young adults, Adv Exp Med Biol, 771 (2012) 51-61.

[11] Walker, P., et al., Public health implications of meat production and consumption, Public Health Nutr, 8 (2005) 348-56.

[12] Djousse, L., et al., Fruit and vegetable consumption and LDL cholesterol: the National Heart, Lung, and Blood Institute Family Heart Study, Am J Clin Nutr, 79 (2004) 213-7.

[13] Higuchi, H., et al., Effects of the Vegetarian Diet on the Blood Rheology of Middle-aged Women [in Japanese], Memoirs of Osaka Kyoiku University II Social Science and Home Economics, 54 (2005) 1-9.

[14] Vitamin B12: Dietary supplementation fact sheet: Office of dietary supplemants. National Instinute of Health. http://ods.od.nih.gov/factsheets/VitaminB12-

HealthProfessional/ (accessed on: 02 May, 2013) (June 2011).

[15] Noncommunicable diseases country profiles 2011. WHO global report in Noncommunicable diseases and mental health 2011, WHO: Genava. p. 209.

[16] Ramaraj, R. and P. Chellappa, Cardiovascular risk in South Asians, Postgrad Med J, 84 (2008) 518-23.

[17] Das, S. K., et al., Nutrition and lipid profile in general population and vegetarian individuals living in rural Bangladesh, Obesity \& Weight Loss Therapy, 2 (2012).

[18] Rice, E.W., Rapid determination of total hemoglobin as hemiglobin cyanide in blood containing carboxyhemoglobin, Clin Chim Acta, 18 (1967) 89-91.

[19] HU, B., H. M, and R. R, International Federation of Clinical Chemistry (IFCC) Scientific Committee, Analytical Section: approved recommendation (1985) on IFCC methods for the measurement of catalytic concentration of enzymes. Part 3. IFCC method for alanine aminotransferase (L-alanine: 2oxoglutarate aminotransferase, EC 2.6.1.2)24: 481-495, J Clin Chem Clin Biochem 24 (1986).

[20] Gindler, E. M. and J. D. King, Rapid colorimetric determination of calcium in biologic fluids with methylthymol blue, Am J Clin Pathol, 58 (1972) 376-82.

[21] Doumas, B. T., W. A. Watson, and H. G. Biggs, Albumin standards and the measurement of serum albumin with bromcresol green, Clin Chim Acta, 31 (1971) 87-96.

[22] Driskell, W. J., et al., Measurement of vitamin A and vitamin $\mathrm{E}$ in human serum by high-performance liquid chromatography, J Chromatogr, 231 (1982) 439-44.

[23] Foster-Swanson A SM, Roberts P, Feld R, Johnson M, S W, et al. (1994) Reference interval Studies of the Rate-Blanked 
Creatinine/Jaffe Method on BM/Hitachi Systems in six U.S Laboratories Clin Chem. Clin Chem 40: 1057

[24] AOAC, AOAC Official Methods of Analysis (1990) 15thed. 2nd Supplement, (1991) 81.

[25] Willett, W. C., 24-Hour Dietary Recall and Food Record Methods. 2 ed. Nutritional Epidemiology, ed. W.C. Willett. 2011: Oxford Scholarship Online.

[26] Wiesmann, D., et al., Explanation of the weighting scheme for the Food Consumption Score (FCS). In:Validation of the world food programme's food consumption score and alternative indicators of household food security. Washington, DC: International Food Policy Research Institute, 10 (2009).

[27] Tohill, B. C., Dietary intake of fruit and vegetables and management of body weight, Centers for Disease Control and Prevention, Alanta, USA, WHO, 2005.

[28] Poian D., A.T. El-Bacha, T. \& Luz, M.R.M.P, Nutrient metabolism in humans: metabolism pathways, Nature Education, 3(9):11, (2010).

[29] Douglas, G., Introduction to Dairy Science and Technology: Milk History, Consumption, Production, and Composition, Dairy Science and Technology, University of Guelph. 2011.
[30] Beta-carotene, University of Maryland Medical Centre, http://www.umm.edu/altmed/articles/beta-carotene- 000286. htm (accessed on 12 May 2013).

[31] A Houlihan, M Pyke, P Scheelings, G Graham, G Eaglesham, T O'Hare, L Wong, P Puwastien and W Jongitsin, Folate content of Asian Vegetables, Australian Government: Rural Industries Research and Development Corporation, 2011.

[32] Human vitamin and mineral requirements, Report of a joint FAO/WHO expert consultation, Bangkok, Thailand, 2002.

[33] Piyathilake, C.J., et al, Local and systemic effects of cigarette smoking on folate and vitamin B-12, Am J Clin Nutr, 60 (1994) 559-66.

[34] Anna, F. L., Keynote Paper: Individual food intake survey methods, National Institute for Food and Nutrition Research, Rome, Italy, http://www.fao.org/docrep/005/Y4249E/y4249e0a. htm (cited on 13.05.2013).

[35] Deriemaeker, P., et al., Nutritional status of Flemish vegetarians compared with non-vegetarians: a matched samples study, Nutrients, 2 (2010) 770-80. 